\title{
Fluid Added-Mass Effect on Flexural Vibration of Hemispherical Shell Structure
}

\author{
Shahrokh Sepehrirahnama, Felix Bob Wijaya, Darren Oon, Eng Teo Ong, Heow Pueh \\ Lee and Kian-Meng Lim
}

Mechanical Engineering Department, National University of Singapore, 9 Engineering Drive 1, Singapore 117575.

\begin{abstract}
(Received 27 February 2019; accepted 2 August 2019)
In this hydroelasticity study, the fluid added-mass effect on a hemispherical shell structure under flexural vibration is investigated. The vibration response of the hemisphere is solved by using a commercial finite element software (ABAQUS) coupled with an in-house boundary element code that models the fluid as potential flow. The fluidstructure interaction is solved as a fully-coupled system by modal superposition to reduce the number of degrees of freedom. The need for an iterative scheme to pass displacement/force information between the two solvers is avoided by direct coupling between the fluid and structure equations. The numerical results on the downward shift in natural frequencies due to added-mass effect compare well with vibration measurements conducted on a stainless-steel bowl with interior and exterior fluid. For water and soap-water solution used in the experiments, the fluid viscosity (varying over a wide range) did not have any significant effect on the wet natural frequencies. This is due to the small viscous boundary layer (milimetre scale) compared to the nominal size of the bowl in centimetres. For such cases, the fluid-added mass only depends on the density of the fluid and the use of potential flow in the numerical model is applicable.
\end{abstract}

\section{INTRODUCTION}

A solid structure moving in contact with a fluid is subjected to additional resistance force. A part of this fluid inertia force is in phase with the motion of the structure, which is associated to the so-called added mass or apparent mass, while the other part is out of phase and associated to added damping. ${ }^{1-3}$ These two parameters are also reported as velocity-coupling and inertiacoupling fluid force coefficients, which relate the added mass and damping to the displaced mass of the fluid by the structure.

Fluid added mass is the key factor in various fields such as underwater energy converters based on flow-induced vibration, ${ }^{4,5}$ design of hydraulic turbine runners ${ }^{3,6,7}$ and dynamic response of floating offshore vessels. ${ }^{1,8,9}$ Although analytical expressions were derived for simple geometries, the added mass estimation for complex geometries requires proper modelling and assumptions. Experimental measurements are ultimately conducted to validate the results of the numerical models. ${ }^{3,6,10,11}$

Analytical studies have provided the added mass values for other shapes such as ellipse, square, circle (2D geometries) and sphere, cube and ellipsoid (3D geometries), but only for the objects in rigid body translation. ${ }^{12}$ For slender bodies such as some designs of ships or submarines, the strip theory is used to calculate the added mass for a thin and narrow section of the exterior surface along the length, and the total added-mass is the sum from all the cross sections. ${ }^{1,9,13}$ However, the strip theory was only valid for rigid body translation of the objects. ${ }^{1}$

The limitations of the analytical methods are overcome by numerical methods such as finite element and boundary element methods for solving added mass problem, especially for cases like flexural vibration of a solid structure, also known as hydroelasticity, with an arbitrary geometry. ${ }^{14-18}$ One way to capture the fluid-solid interaction was to integrate finite element and boundary element methods into a fully coupled numerical method at the formulation level. ${ }^{7,19,20}$ However, this method is computationally expensive since the augmented system matrices such as stiffness matrix are no longer sparse ma- trices and become larger than matrices of each method individually.

The alternate approach, known as partitioned iterative solution, is to use decoupled numerical algorithms by transferring data back and forth between the structure vibration solver (finite element method) and the fluid flow solver (boundary element method). ${ }^{21,22}$ In this iterative algorithm, ${ }^{22}$ the termination criterion is convergent frequency for free vibration. Despite being straight forward and easy to implement, the iterative algorithms are computationally expensive, and their convergence is not guaranteed. ${ }^{21,22}$

To avoid the numerical challenges of both the fully coupled FEM-BEM method and the iterative algorithm, the modal superposition was applied. ${ }^{14,15}$ For a structure in contact with a fluid, the superposition principle allows the fluid loading contributions from various dry mode shapes to be added up to give the total fluid effect. Writing the mode shapes of the wet structure as a linear combination of a subset of dry mode shapes, the fluid-structure interaction algorithm does not need any iteration and the total number of degrees of freedom is also reduced.

This study aims to calculate the fluid inertia effects in terms of added mass on a hemispherical shell (open curved shell) undergoing flexural vibration. The fluid inertia effect is reported as the downward shift of the wet natural frequencies from the original dry natural frequencies. The numerical algorithm uses a commercial finite element software for the structural vibration analysis and an in-house boundary element code for the fluid loading. A fully-coupled system of equations are formed for the fluid-structure interaction by the superposition of the selected dry mode shapes, reducing the number of degrees of freedom and providing accurate results for the leading wet natural frequencies and mode shapes. For experimental validation, vibration measurements are conducted on a steel bowl under two loading configurations: partially immersed in a tank of water (exterior fluid loading or Test (A)) and partially filled with fluid (interior fluid loading or Test (B)). 


\section{THEORETICAL FORMULATION}

\subsection{Modal Superposition}

The vibration equation of a structure subjected to fluid loading is given by:

$$
[m]\{\ddot{\mathbf{u}}\}+[K]\{\mathbf{u}\}=\left\{\mathbf{f}_{\mathbf{l}}\right\}+\left\{\mathbf{f}_{\mathbf{e}}\right\}
$$

where $[m]$ and $[K]$ are the structural mass and stiffness matrices, respectively, $\{\mathbf{u}\}$ is the displacement vector, $\left\{\mathbf{f}_{\mathbf{l}}\right\}$ represents the force exerted on the solid structure due to the fluid loading, and $\left\{\mathbf{f}_{\mathbf{e}}\right\}$ is any other external loading. For free vibration analysis, the external loading $\left\{\mathbf{f}_{\mathbf{e}}\right\}$ is taken to be zero. For a discretized finite element model, the structural matrices are square with size $q$ corresponding to the total number of degrees of freedom.

First, we consider the dry structure without the fluid loading in equation (1) and solve for its free vibration response $\{\mathbf{u}\}=$ $\{\mathbf{d}\} \exp ^{-i \omega t}$ :

$$
\left(-\omega^{2}[m]+[K]\right)\{\mathbf{d}\}=\{\mathbf{0}\}
$$

where $\omega$ and $\{\mathbf{d}\}$ are the dry-mode natural frequency and mode shape, respectively. This equation has $q$ number of solutions with $\omega_{i}$ and $\{\mathbf{d}\}_{i}$ for the $i$ th mode of vibration. Using modal superposition, the structure response $\{\mathbf{u}\}$ in equation (1) can be approximated by:

$$
\{\mathbf{u}\}=\sum_{j=1}^{Q} L_{j}\{\mathbf{d}\}_{j}=[D]\{L\} ;
$$

where $[D]=\left[\{\mathbf{d}\}_{1},\{\mathbf{d}\}_{2}, \cdots,\{\mathbf{d}\}_{Q}\right]$ is the modal matrix with $Q$ the number of selected mode shapes, and $\{L\}$ is the vector of principal coordinates which contains the contribution factor of each and every dry mode-shape in the response of the structure to a given external loading.

For each dry mode shape $\{\mathbf{d}\}_{j}$, the fluid loading $\{\overline{\mathbf{f}}\}_{j}$ on the structure is calculated using a boundary element code (described in the next section). By applying the modal superposition, the total fluid force $\left\{\mathbf{f}_{\mathbf{l}}\right\}$ acting on the structure with displacement profile given by Equation (3) is:

$$
\left\{\mathbf{f}_{\mathbf{l}}\right\}=\sum_{j=1}^{Q} L_{j}\{\overline{\mathbf{f}}\}_{j}=[F]\{L\},
$$

where $[F]=\left[\{\overline{\mathbf{f}}\}_{1},\{\overline{\mathbf{f}}\}_{2}, \cdots\{\overline{\mathbf{f}}\}_{Q},\right]$ is the interaction force matrix (similar to $[D]$ ) that is formed by the assembly of the terms $\{\overline{\mathbf{f}}\}_{j}$.

By substituting equations (3) and (4) into (1) and premultiplying equation (1) by the transpose of the modal matrix $[D]^{T}$, one can write a strongly-coupled but reduced system of equations:

$$
[D]^{T}[m][D]\{\ddot{L}\}+[D]^{T}[K][D]\{L\}=[D]^{T}[F]\{L\} .
$$

It is noted that the fluid-structure force matrix $[F]$ depends on the dry mode shapes of the structure, and the unknowns in the above equation are the principal coordinates $\{L\}$ in terms of the dry mode shapes.

\subsection{Potential Flow Theory}

To model the fluid loading on the structure, the potential flow theory is used to account for the mass density of the fluid.

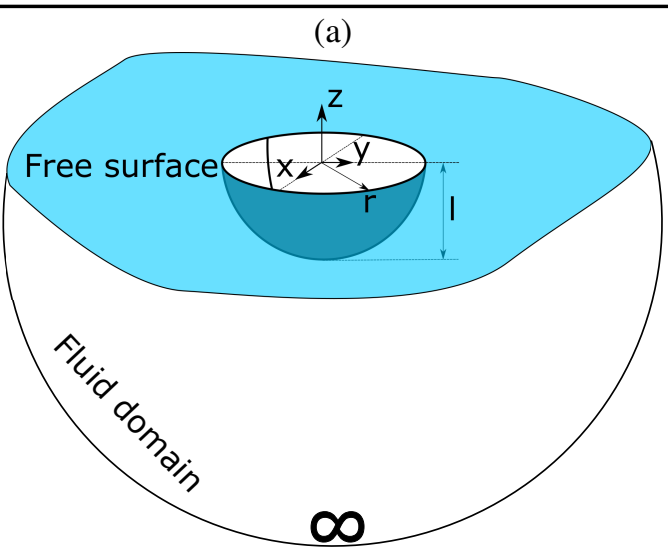

(a)

(b)

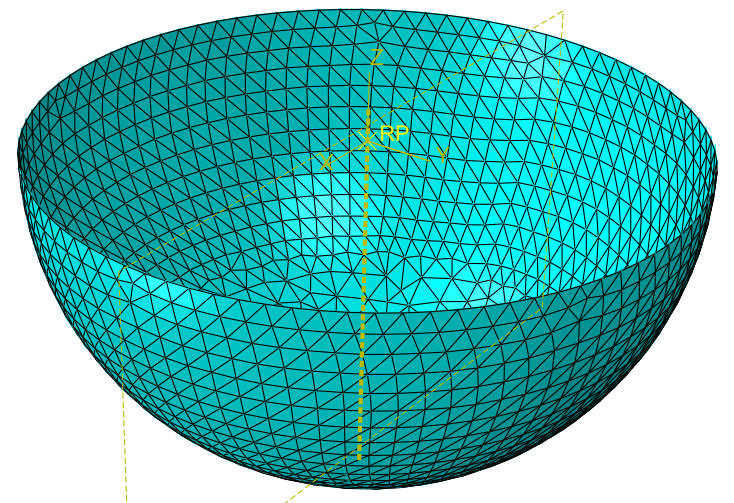

Figure 1. (a)Floating hemispherical shell in a semi-infinite fluid domain and (b) its discretized model of $10 \mathrm{~cm}$ radius $r$ an thickness $t . l$ is the immersion depth.

The solid structure is assumed to be partially submerged in a semi-infinite fluid domain, as shown in Fig. 1, and the vibrational motion of the structure interacts with fluid domain at its interface. The governing equation for potential flow, neglecting the fluid viscosity, is given by:

$$
\begin{gathered}
\nabla^{2} \phi=0 ; \\
p=-\rho \dot{\phi}+\rho g z ;
\end{gathered}
$$

where $\phi$ is the velocity potential and $\mathbf{v}=\nabla \phi, p$ is the total dynamic pressure, $\rho$ is the fluid density and $g$ is the gravity acceleration constant. Equation (7) is known as linearized Bernoulli's equation and derived from the momentum conservation equation. The first term on the right-hand side of the Bernoulli's equation is to account for the oscillation of the interface about its mean position whereas the second term is associated to the change in the buoyancy force. By writing $\phi=\Phi e^{-i \omega t}$, equation (6) is solved for the amplitude of the velocity potential $\Phi$ with the following boundary conditions: (i) prescribed surface velocity for the $j$ th mode shape at the fluid-structure interface, (ii) vanishing fluid velocity at infinity, and (iii) zero dynamic pressure at the free surface of the fluid. It is convenient to introduce a new variable $\hat{\Phi}$ for the displacement potential such that $-i \omega \hat{\Phi}=\Phi$. This gives:

$$
\frac{\partial \hat{\Phi}}{\partial \mathbf{n}}=\mathbf{d}_{j} \cdot \mathbf{n}
$$


where $\mathbf{n}$ is the unit normal vector to the interface between the fluid and structure (pointing away from the fluid), and $\mathbf{d}_{j}$ is the displacement of the interface. Equation (6) can be written in the form of boundary integral equation as follow:

$$
\begin{gathered}
c \hat{\Phi}\left(\mathbf{x}_{t}\right)+\int_{\Gamma} \hat{\Phi}\left(\mathbf{x}_{s}\right) \frac{\partial G\left(\mathbf{x}_{t} ; \mathbf{x}_{s}\right)}{\partial n} d \Gamma= \\
\int_{\Gamma} \frac{\partial \hat{\Phi}\left(\mathbf{x}_{s}\right)}{\partial n} G\left(\mathbf{x}_{t} ; \mathbf{x}_{s}\right) d \Gamma
\end{gathered}
$$

where $c$ is the solid angle which depends on whether the target point is inside the domain or on the boundary, and $\Gamma$ is the wet interface surface. The second and third boundary conditions are taken care of by choosing the proper Green's function:

$$
G\left(\mathbf{x}_{t} ; \mathbf{x}_{s}\right)=\frac{1}{4 \pi\left|\mathbf{x}_{t}-\mathbf{x}_{s}\right|}-\frac{1}{4 \pi\left|\mathbf{x}_{t}-\overline{\mathbf{x}_{s}}\right|} ;
$$

where $\mathbf{x}_{t}$ is the position vector of the target point, $\mathbf{x}_{s}$ and $\overline{\mathbf{x}_{s}}$ are the position vectors of a boundary point and its image with respect to the free-surface plane, respectively.

The flexural vibration of the structure is solved at the static equilibrium state at which the buoyancy force balances its weight. Displacement in the vertical direction $\mathrm{z}$ results in harmonic changes of the buoyancy force, also known as buoyancy spring. Without loss of generality, this buoyancy spring that affects only translational motion can be neglected when we are solving for the flexural modes. The buoyancy spring stiffness only contributes to the total stiffness of the system involving rigid body motion due to changes in the hydrostatic pressure.

After solving for $\hat{\Phi}$, one can write the pressure as:

$$
p=-i \omega \rho \Phi e^{-i \omega t}=-\omega^{2} \rho \hat{\Phi} e^{-i \omega t} .
$$

For the $j$ th mode, the surface force at node $k$, calculated from dynamic pressure, is:

$$
\mathbf{f}_{j}^{(k)}=\omega^{2} \rho \hat{\Phi}^{(k)} \mathbf{n}^{(k)} \Gamma^{(k)}
$$

where $\Gamma^{(k)}$ is the area of the fluid element at node $k$. The force is only calculated for nodes on the wet interface and is zero at other nodes. By Newton's third law, the interaction force vector acting on the structure for the $j$ th mode is:

$$
\begin{array}{r}
\{\overline{\mathbf{f}}\}_{j}=-\omega^{2} \rho\left[\hat{\Phi}^{(1)} \mathbf{n}^{(1)} \Gamma^{(1)}, \cdots, \hat{\Phi}^{(s)} \mathbf{n}^{(s)} \Gamma^{(s)}\right]^{T}= \\
\omega^{2} \rho\left\{P_{j}\right\}
\end{array}
$$

where $\left\{P_{j}\right\}$ is the vector containing of the force contribution $\left(-\hat{\Phi}^{(k)} \mathbf{n}^{(k)} \Gamma^{(k)}\right)$ at each element $k$ for mode $j$.

\subsection{Wet Natural Frequency}

After computing $\{\overline{\mathbf{f}}\}_{j}$ for all the $Q$ dry mode shapes, equation (5) can be rewritten as:

$$
\begin{array}{r}
\left(-\omega^{2}[D]^{T}[m][D]-\omega^{2} \rho[D]^{T}\left[\left\{P_{1}\right\}, \cdots,\left\{P_{Q}\right\}\right]\right. \\
\left.+[D]^{T}[K][D]\right)\{L\}=\{0\}
\end{array}
$$

The modal added mass matrix is thus given by:

$$
\left[M_{a}\right]=\rho[D]^{T}\left[\left\{P_{1}\right\},\left\{P_{2}\right\}, \cdots,\left\{P_{Q}\right\}\right]
$$

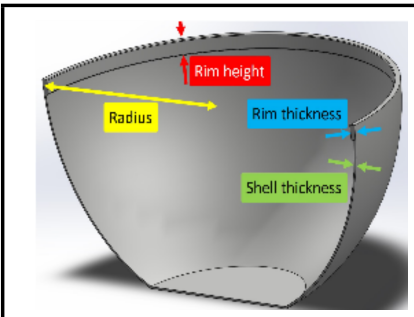

(a)

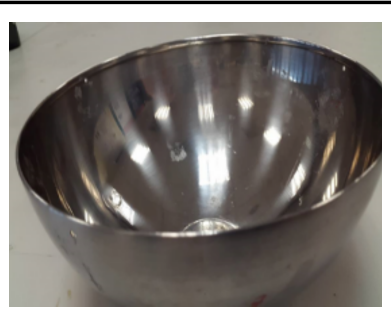

(b)
Figure 2. Hemispherical test specimen and its dimensions.

resulting in the final system of equations to solve for the wetmode frequency $\hat{\omega}$ :

$$
\left(-\hat{\omega}^{2}[\bar{M}]-\hat{\omega}^{2}\left[M_{a}\right]+[\bar{K}]\right)\{L\}=\{0\} .
$$

Here, $[\bar{M}]=[D]^{T}[m][D]$ is the modal structural mass, and $[\bar{K}]=[D]^{T}[K][D]$ is the modal structural stiffness. After $\hat{\omega}_{j}$ and $\{L\}_{j}$ are obtained, the $j$ th wet mode shape can be obtained by:

$$
\{\hat{\mathbf{d}}\}_{j}=[D]\{L\}_{j} .
$$

\subsection{Computational Aspects}

According to the modal superposition theory, the selected dry mode shapes can be viewed as shape functions to approximate the vibration response of the system, since they are linearly independent functions. By keeping these shape functions throughout the numerical analysis, there is no need for iterations as used in partitioned iterative solvers. ${ }^{22}$ The accuracy of this method is studied by increasing the number of selected dry modes for the modal superposition.

In this algorithm, the in-vacu free vibration of the structure was performed by the commercial FEM package, ABAQUS 6.14. The mass and stiffness matrices and the mode shapes for the entire model and the wet subset of nodes are extracted out for solving the free vibration of the wet structure. After the fluid loading is calculated from the boundary element solver, the wet natural frequencies and mode shapes are obtained simultaneously. The size of the matrices in equation (16) is the same as the number of selected modes, which is much smaller than the number of the nodes of the discretized structure. Hence, there is a tremendous speed-up in solving the eigenvalue problem. The calculation of fluid pressure for each mode shape is the most time consuming; however, it can be done in parallel for all the selected dry modes to reduce the overall computation time.

\section{EXPERIMENTAL VALIDATION}

To validate the numerical model, an experiment was conducted on a hemisphere bowl made of stainless steel (Fig. 2). The bowl has a flat bottom and its radius varies from $0.11 \mathrm{~m}$ (near the bottom surface) to $0.10 \mathrm{~m}$ at the rim. The thickness of the bowl, which is denoted by shell thickness in Fig. 2, varies from $0.5 \mathrm{~mm}$ (just below the rim) to $1.00 \mathrm{~mm}$ (at the bottom surface of the bowl). From the measurement at 25 different points along the circumference, the minimum, mean, and maximum values of the rim thickness are $1.65 \mathrm{~mm}, 1.72 \mathrm{~mm}$, and $1.85 \mathrm{~mm}$, respectively. By considering the mass of the bowl and volume of the material, the rim thickness was estimated to be $1.6 \mathrm{~mm}$, which is close to the minimum measured value. The rim height was found to be approximately $5 \mathrm{~mm}$. 


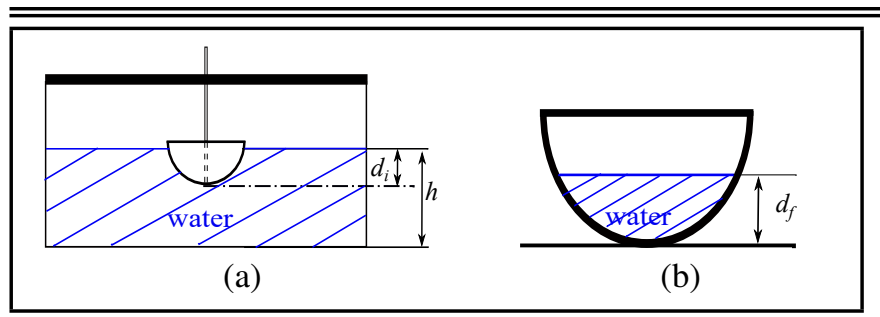

Figure 3. Schematics of setups showing (a) immersion depth $d_{i}$ and water height inside the tank $h$ in Test (A) and (b) fluid depth $d_{f}$ in Test (B). The height of the bowl is $9 \mathrm{~cm}$.

Measurement of natural frequencies of the bowl was performed in two configurations (schematics shown in Fig. 3). In Test (A), the bowl was hung from an aluminium bar and immersed in water contained in a large tank. The purpose of this measurement is to study the added mass effect induced by an exterior fluid. In Test (B), the bowl was fixed at its bottom surface and filled in turn with water and a soap-water solution. Both fluids have the same mass density but significantly different dynamic viscosity. The soap-water solution has a viscosity of $10 \mathrm{dPa} \cdot \mathrm{s}$, which is about thousand times larger than that of water. The purpose of this measurement is to study the added mass effect induced by an interior fluid and investigate any significant effect of fluid viscosity on the added mass effect.

Figure 3 shows the schematics of the setups and the parameters that were varied in the experiments. For the first configuration, two parameters, namely immersion depth $\left(d_{i}\right)$ and water height $(h)$, were varied. Four values of $d_{i}(4.5,5.0,6.5$, and $7.5 \mathrm{~cm})$ and four values of $h(17,25,33$, and $41 \mathrm{~cm})$ were used in the measurement. For the second configuration, the fluid depth $\left(d_{f}\right)$ was varied. Similar to the first configuration, four values of $d_{f}(4.5,5.0,6.5$, and $7.5 \mathrm{~cm})$ were used.

For both Tests (A) and (B), an impact force, via a sharp knock that excites frequencies up to $3000 \mathrm{~Hz}$, was exerted in the horizontal plane along the radial direction at a point on the rim of the bowl. The vibration response of a point on the bowl near the rim was measured by using a Polytec's PDV 100 portable digital vibrometer. The setting parameters for the vibrometer are as follows: frequency span $=2000 \mathrm{~Hz}$, sampling frequency $=5120 \mathrm{~Hz}$, frequency resolution $=0.15625 \mathrm{~Hz}$, window function $=$ exponential, and number of averages $=10$. For each round of the test, the bowl was hit 10 times and the recorded responses were averaged out by the software of the vibrometer. Before each round of test, it was ensured that the water free-surface was settled and there was no additional movement in the setup. The experiment was repeated five to six rounds to obtain the mean, maximum and minimum values of measured frequencies. The error bound of the experimental results was estimated to be $\pm 10 \%$.

From frequency spectrum of the vibration response, the natural frequencies of the bowl were identified by the observed peaks in the spectrum. This procedure was repeated for all fluid levels illustrated in Fig. 3.

\section{RESULTS}

The numerical results are compared against the experimental results of the stainless-steel bowl. In the numerical simulations, the density and Young's modulus of the hemispherical shell are taken as $\rho_{s}=7800 \mathrm{~kg} / \mathrm{m}^{3}$ and $E_{s}=210 \mathrm{GPa}$, respectively. The density of the fluid is $\rho=1000 \mathrm{~kg} / \mathrm{m}^{3}$. The structural vibration analysis was performed using the finite element software (ABAQUS 6.14), and linear shell elements (S4) with six degrees of freedom at each node were used. Conver-

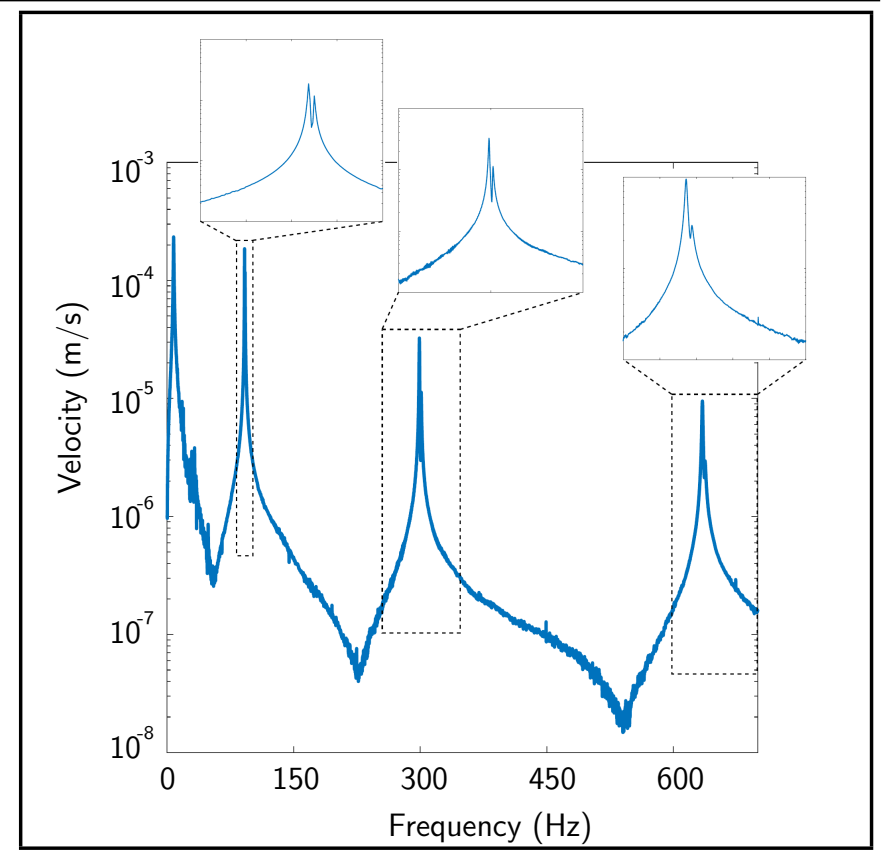

Figure 4. Averaged frequency spectrum of the bowl's vibration response.

gence tests were carried out to obtain a suitable fine mesh for accurate results. For potential flow simulation, boundary elements were chosen to be constant panels with linear geometry. The finite element and boundary element models share a common interface mesh so that surface displacement is transferred easily between the two solvers.

\subsection{Dry Natural Frequency}

The dry natural frequencies of the bowl were first measured and then compared with the results from the finite element model for benchmarking. For this set of measurements, the bowl was placed on a very soft sponge (negligible stiffness) to provide as close to a free boundary condition at the rim as possible. The averaged frequency spectrum of the bowl's vibration response is shown in Fig. 4.

Four peaks are observed from 0 to $700 \mathrm{~Hz}$ in in the frequency spectrum, as shown in Fig. 4. From the magnified views shown in Fig. 4, it can be seen that there are actually two peaks separated by a small gap at the frequencies $92 \mathrm{~Hz}, 300 \mathrm{~Hz}$, and $635 \mathrm{~Hz}$. These are repeated natural frequencies due to the axisymmetric nature of the bowl. Therefore, the first seven natural frequencies of the bowl were identified. The last six frequencies are associated with bowl's flexural mode shapes while the first frequency correspond to the rigid body motion of the bowl bouncing on the sponge support (as shown later in Table 1).

To confirm the frequencies associated with the flexural mode shapes, numerical simulation of the free vibration of the bowl was conducted using ABAQUS 6.14. Figure 5a shows the finite element model of the bowl which was obtained by 3D scanning. The bowl is approximately a hemisphere (radius $r \approx 0.1 \mathrm{~m}$ ) with a flat bottom (height $\approx 0.09 \mathrm{~m}$ ). The red region indicates the rim of the bowl which is modelled with three different values of thickness $\left(t_{r}\right)$, namely $1.65,1.72$, and $1.85 \mathrm{~mm}$ to correspond to the minimum, mean, and maximum values of the rim thickness obtained from measurements. The thickness of other region of the bowl was set to vary linearly from $0.5 \mathrm{~mm}$ to $1.0 \mathrm{~mm}$. Stress free boundary condition was imposed to simulate a free vibration behavior. The first six natural frequencies corresponding to the flexural mode shapes of 


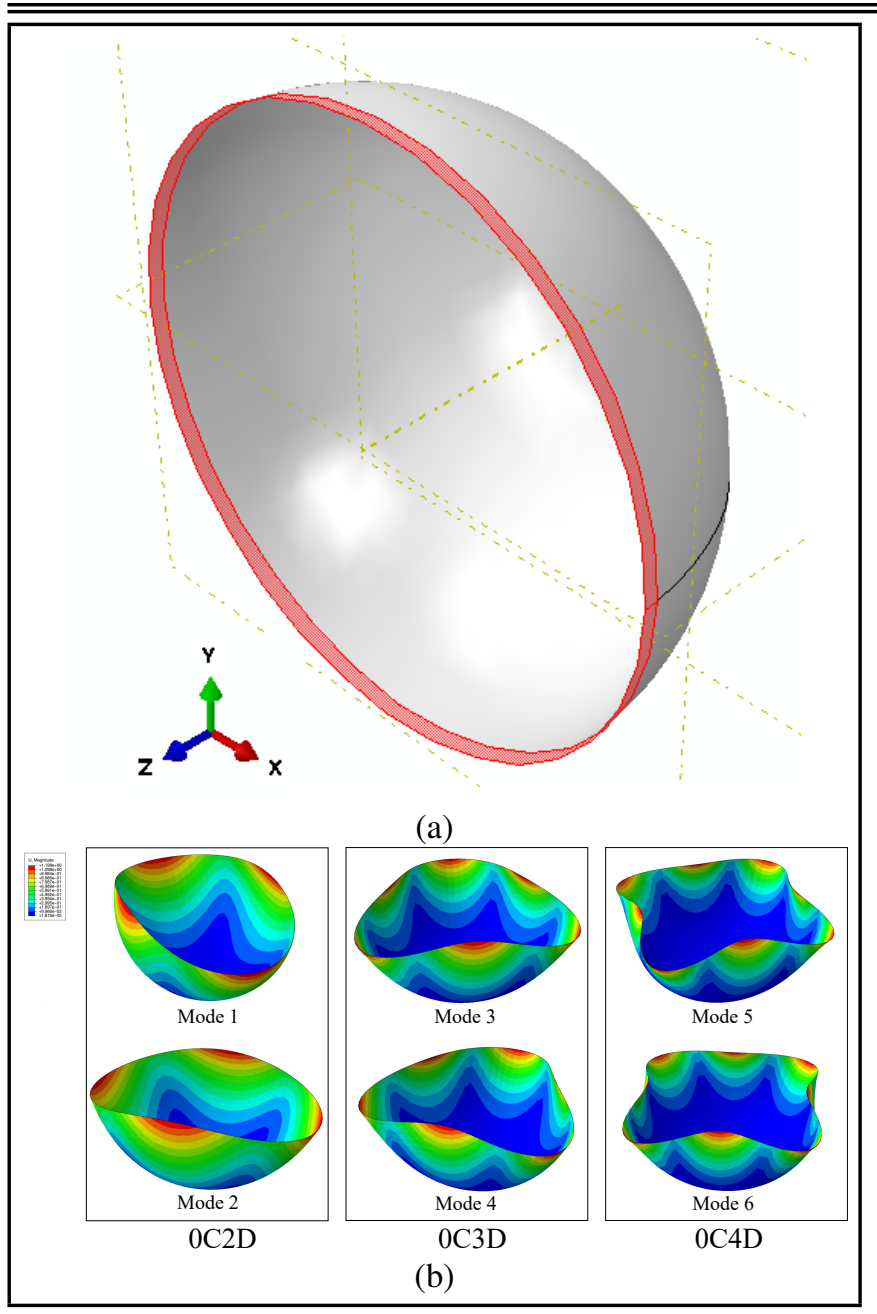

Figure 5. (a) Finite element model of the bowl. (b) Flexural mode shapes of the bowl obtained from the simulation. The mode shapes are paired due to the symmetry of the geometry used in the simulation.

the bowl (as shown in Fig. 5b) were obtained in the frequency range of 0 to $700 \mathrm{~Hz}$. It is again noted that the repeated natural frequencies and mode shapes are due to the nearly symmetric geometry of the bowl. For mode 1 and mode 2, there is no nodal circle and two nodal diameters; this pair of modes are referred to as mode 0C2D. Similarly, modes 3 and 4 are referred to as mode 0C3D (with three diameter nodal lines) and modes 5 and 6 as mode 0C4D (with four diameter lines). The values of the repeated frequencies obtained from simulation are tabulated together with the experiment data obtained from the experimental measurements of the bowl in Table 1.

From this table, it can be deduced that the first natural frequency obtained from the experiments corresponds to the rigid body motion of the bowl on the sponge since this is not accounted for in the numerical simulation. For the other six frequencies, the simulation results are relatively close to the experiment data. The relative difference with respect to the experimental values is found to be less than $15 \%$ for all the cases, except the frequencies of mode 2 and mode 3 for the case of $t_{r}=1.85 \mathrm{~mm}$.

\subsection{Wet Natural Frequency with Exterior Fluid}

The wet natural frequencies for the bowl partially immersed in water (first configuration) were measured following the same procedure described earlier. Figure 6 shows the frequency spectra of the bowl's vibration response for different

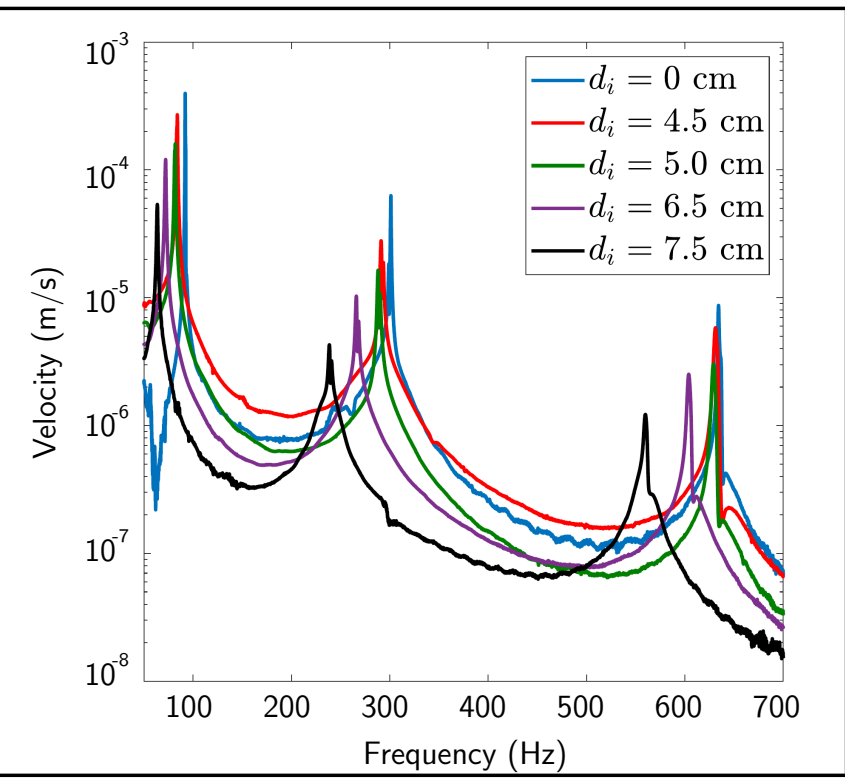

Figure 6. Frequency spectra of the bowl's vibration response measured for different values of $d_{i}$ and $h=41 \mathrm{~cm}$ for exterior fluid configuration.

values of $d_{i}$ and $h=41 \mathrm{~cm}$. The frequency range of the spectrum excludes the resonance peaks associated with rigid body pendulum mode below $50 \mathrm{~Hz}$ and only shows the peaks corresponding to the flexural vibration. As expected, the peaks shift to the left as the immersion depth increases. This indicates the decrease of the bowl's natural frequencies which reflects the effect of fluid added mass on the bowl vibration.

To better illustrate the frequency downshift observed in Fig. 6, the numerical model was used to calculate the corresponding wet natural frequencies and compared with the experimental data for different values of $d_{i}$ (Fig. 7). As seen in the figure, the numerical results are in good agreement with the experiment data, with the largest relative difference being less than $15 \%$. This reflects the accuracy of the numerical model in predicting wet natural frequencies of a structure in contact with a fluid. Both the numerical results and experiment data show that the frequency downshift increases as immersion depth $d_{i}$ increases.

\subsection{Wet Natural Frequency with Interior Fluid}

The natural frequencies of the bowl with interior fluid (Test (B)) are reported in this section. Two fluids (water and soapwater) of similar mass densities but significantly different viscosities were used in turn. Figure 8 shows the frequency spectra of bowl's vibration response measured for fluid filled to different levels $d_{f}$. Similar to the case of exterior fluid, the peaks indicating natural frequencies of the system shift to the left as the fluid level $\left(d_{f}\right)$ increases for both water and soap-water solution.

The wet natural frequencies for water as the interior fluid are compared with the measurements obtained for Test (A) (Table 2). It can be seen that the two sets of wet natural frequencies are relatively close, since the largest relative difference between the results of Tests $(\mathrm{A})$ and $(\mathrm{B})$ is less than $10 \%$, when $d_{f}=d_{i}$. This indicates that the fluid added mass effects in both configurations are the same when the bowl's wetted area is kept the same despite the large difference in the volume of water used in both configurations. This behaviour is also predicted by the numerical model as the same fluid loading acts on the bowl for both the internal and external fluid when the 
Table 1. Natural frequencies of the bowl obtained from both simulation and experiment on a sponge.

\begin{tabular}{||c|ccccccc||}
\hline \multirow{2}{*}{ Method } & \multicolumn{7}{|c||}{ Natural frequencies $(\mathrm{Hz})$} \\
\cline { 2 - 8 } & 1 & 2 & 3 & 4 & 5 & 6 & 7 \\
\hline Simulation $\left(t_{r}=1.65 \mathrm{~mm}\right)$ & - & 99.42 & 99.42 & 282.97 & 282.98 & 550.79 & 550.83 \\
Simulation $\left(t_{r}=1.72 \mathrm{~mm}\right)$ & - & 102.36 & 102.38 & 293.43 & 293.46 & 572.07 & 572.11 \\
Simulation $\left(t_{r}=1.85 \mathrm{~mm}\right)$ & - & 107.76 & 107.78 & 312.00 & 312.03 & 608.91 & 608.96 \\
Bowl on sponge & 8.02 & 91.88 & 92.50 & 299.06 & 301.09 & 634.29 & 637.50 \\
\hline
\end{tabular}

Table 2. Wet natural frequencies of the bowl measured in both configurations 1 and 2 when it was in contact with water.

\begin{tabular}{||c|c|ccc||}
\hline \multirow{2}{*}{$d_{f}=d_{i}$} & \multirow{2}{*}{ Configuration } & \multicolumn{3}{|c|}{ Natural frequencies (Hz) } \\
\cline { 3 - 5 } & & 0C2D & 0C3D & 0C4D \\
\hline \multirow{2}{*}{$4.5 \mathrm{~cm}$} & Test (A) & 84.22 & 293.25 & 633.25 \\
& Test (B) & 85.00 & 293.70 & 632.90 \\
\hline \multirow{2}{*}{$5.0 \mathrm{~cm}$} & Test (A) & 80.31 & 286.82 & 627.95 \\
& Test (B) & 81.82 & 288.78 & 629.17 \\
\cline { 2 - 5 } & Test (A) & 70.63 & 264.22 & 600.40 \\
\multirow{2}{*}{$6.5 \mathrm{~cm}$} & Test (B) & 68.96 & 259.45 & 594.57 \\
\cline { 2 - 5 } & Test (A) & 63.38 & 239.35 & 559.24 \\
\multirow{2}{*}{$7.5 \mathrm{~cm}$} & Test (B) & 59.22 & 225.74 & 535.70 \\
\cline { 2 - 5 } & \multicolumn{4}{|c}{} \\
\hline
\end{tabular}

Table 3. Natural frequencies of the bowl for different values of $d_{f}$.

\begin{tabular}{||c|c|ccc||}
\hline \multirow{2}{*}{$d_{f}(\mathrm{~cm})$} & \multirow{2}{*}{ Fluid } & \multicolumn{3}{|c|}{ Natural frequencies $(\mathrm{Hz})$} \\
\cline { 2 - 5 } & & 0C2D & 0C3D & 0C4D \\
\hline \multirow{2}{*}{4.5} & Water & 84.69 & 293.85 & 632.50 \\
& Soap-Water & 83.59 & 291.95 & 631.40 \\
\hline \multirow{2}{*}{5.0} & Water & 81.72 & 288.55 & 628.90 \\
& Soap-Water & 80.78 & 287.45 & 627.80 \\
\hline \multirow{2}{*}{6.5} & Water & 69.06 & 259.45 & 594.70 \\
\cline { 2 - 4 } & Soap-Water & 67.81 & 256.00 & 589.50 \\
\hline \multirow{2}{*}{7.5} & Water & 59.22 & 225.70 & 535.80 \\
& Soap-Water & 59.53 & 226.10 & 538.80 \\
\hline
\end{tabular}

wetted area is the same. Figure 9 shows a comparison of the experimental data with the numerical simulation results for internal fluid. Both experimental measurements using water and soap-water give data that are close to the natural frequencies predicted by the numerical model, with the largest relative difference being less than $17 \%$. It is noted that the numerical simulation results are exactly the same for internal and external fluid.

In Table 3, the natural frequencies of the bowl filled with water are compared with those measured when the bowl was filled with soap-water solution. The frequencies measured in both cases are very close to each other, despite the soap-water having a viscosity a thousand times that of water; the largest relative difference between the two sets of results is less than $2 \%$. This confirms that the wet natural frequencies are only dependent on the fluid added-mass or fluid density. The fluid viscosity has insignificant effects on the measured wet natural frequencies. The good agreement between the experimental and numerical results in Fig. 9 also confirms the negligible effect of fluid viscosity when predicting the added mass effect of the fluid.

The negligible effect of viscosity in this study can be explained by the small length scale of the boundary layer in the fluid, which is of the order $\mathcal{O}(\sqrt{\nu / \omega})$ where $\nu$ is the kinematic viscosity of the fluid. For the present study, the viscous boundary layer length-scale ranges from 0.1 to $1 \mathrm{~mm}$ for the extreme case of frequency down to $100 \mathrm{~Hz}$ and viscosity up to $10^{-3} \mathrm{~m}^{2} / \mathrm{s}$ for the soap-water solution. Compared to the centimetre length-scale of the hemispherical bowl, the boundary layer is too small to have any significant influence on the wet frequencies of the bowl. Hence, for structures in the lengthscale of centimetres or larger, such as ships or other offshore marine structures, the use of potential flow is applicable to model the fluid added-mass effect, and subsequently wet natural frequencies.

\section{CONCLUSION}

The effect of fluid loading on a vibrating hemispherical shell has been studied both theoretically and experimentally. The proposed numerical scheme uses the modal superposition method to apply direct coupling between the structural and fluid solvers, avoiding the need for iterations in passing the displacement and force information between the two solvers. The numerical scheme provides accurate results for the wet natural frequencies and mode shapes. Experiments were conducted for two configurations of a steel bowl (with exterior fluid and interior fluid) to validate the numerical results. It was also shown that the wet natural frequencies, being dependent only on the fluid added-mass, are not affected by drastic change in viscosity of the fluid. This is due to the small viscous boundary layer compared to the nominal size of the structure. Hence, the potential flow is an applicable model for predicting the wet natural frequencies and fluid added-mass effects for vibrating structures interacting with a fluid medium in such cases.

\section{ACKNOWLEDGMENT}

This study is supported by the research project "Advanced Computational Tools for Fast Analysis and Design of Ships against Vibration and Noise" funded by the Singapore Maritime Institute (Project ID: SMI-2015-MA-08).

\section{REFERENCES}

1 Newman, J. N. The theory of ship motions. Advances in applied mechanics, 18, 221-283, 1979. https://dx.doi.org/10.1016/S0065-2156(08)70268-0.

2 Rahman, M. and Bhatta, D. D. Evaluation of added mass and damping coefficient of an oscillating circular cylinder. Applied Mathematical Modelling, 17(2), 70-79, 1993. https://dx.doi.org/10.1016/0307-904X(93)90095-X.

3 Valentín, D., Presas, A., Egusquiza, E., and Valero, C. Experimental study on the added mass and damping of a disk submerged in a partially fluidfilled tank with small radial confinement. Journal of Fluids and Structures, 50, 1-17, 2014. https://dx.doi.org/10.1016/j.jfluidstructs.2014.06.006.

4 Vikestad, K., Vandiver, J. K., and Larsen, C. M. Added mass and oscillation frequency for a circular cylinder subjected to vortex-induced vibrations and external disturbance. Journal of Fluids and Structures, 14(7), 1071-1088, 2000. https://dx.doi.org/10.1006/jfls.2000.0308.

5 Arionfard, H. and Nishi, Y. Experimental investigation of a drag assisted vortex-induced vibration energy converter. Journal of Fluids and Structures, 68, 48-57, 2017. https://dx.doi.org/10.1016/j.jfluidstructs.2016.10.002.

6 Rodriguez, C. G., Egusquiza, E., Escaler, X., Liang, Q. W., and Avellan, F. Experimental investigation of added mass effects on a Francis turbine runner in still water. 


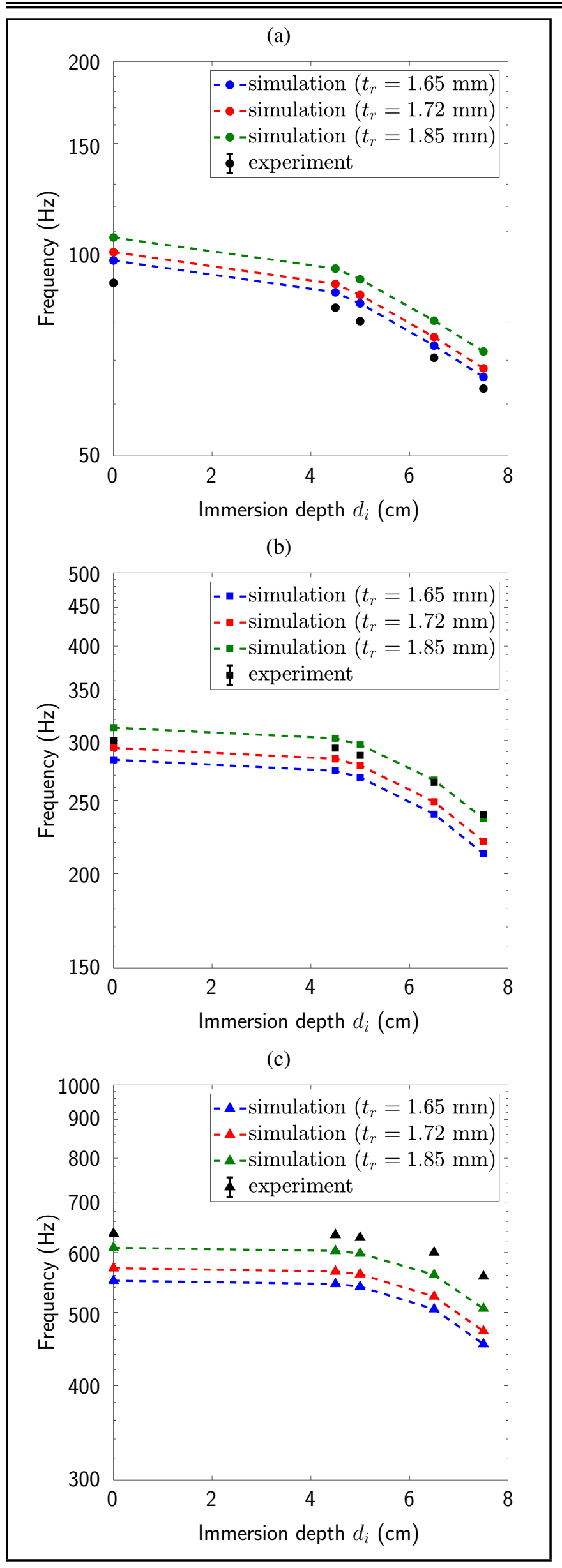

Figure 7. Wet natural frequencies of (a) mode 0C2D, (b) mode 0C3D, and (c) mode 0C4D for different values of $d_{i}$ and $h=41 \mathrm{~cm}$. (a)

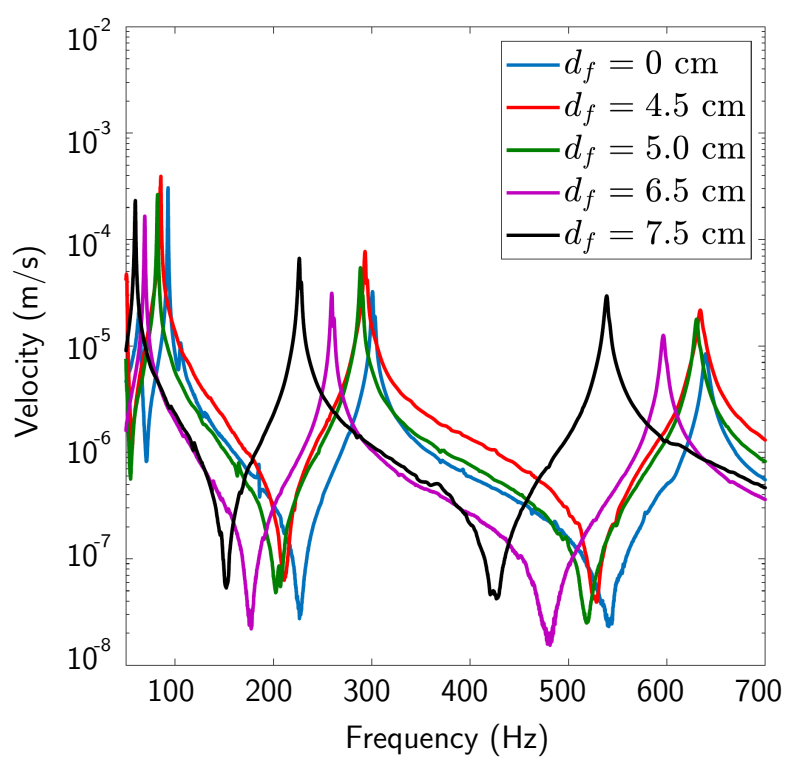

(b)

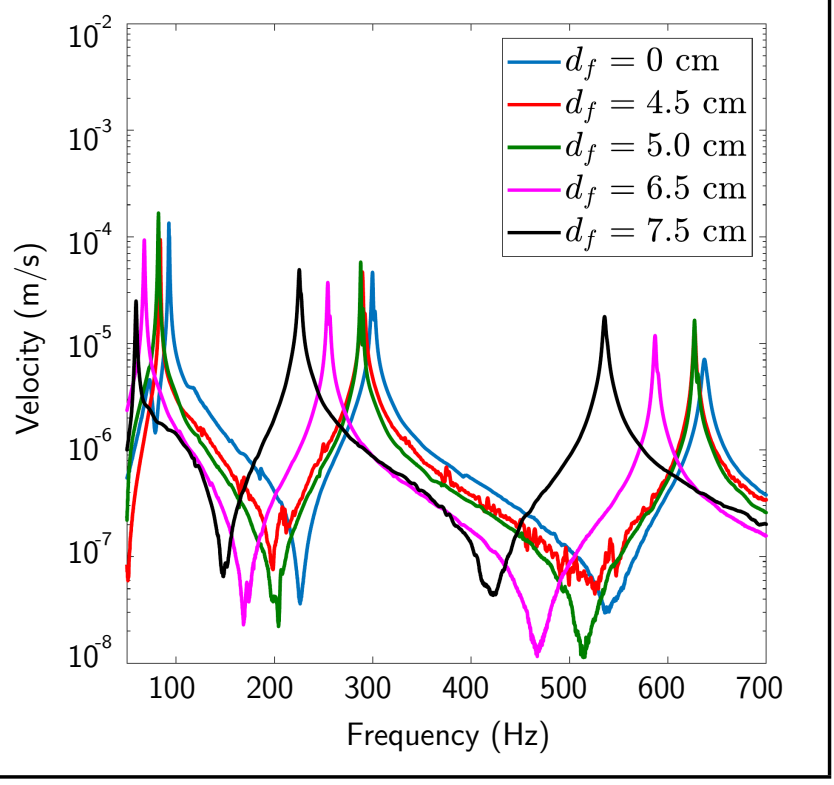

Figure 8. Frequency spectra of vibration response of the bowl for different values of $d_{f}$ with (a) water and (b) soap-water solution as the internal fluid.

Journal of Fluids and Structures, 22(5), 699-712, 2006. https://dx.doi.org/10.1016/j.jfluidstructs.2006.04.001.

7 Liang, Q. W., Rodríguez, C. G., Egusquiza, E., Escaler, X., Farhat, M., and Avellan, F. Numerical simulation of fluid added mass effect on a francis turbine runner. Computers \& Fluids, 36(6), 1106-1118, 2007. https://dx.doi.org/10.1016/j.compfluid.2006.08.007.

$8 \mathrm{Wu}$, J. S. and Hsieh, M. An experimental method for determining the frequency-dependent added mass and added mass moment of inertia for a floating body in heave and pitch motions. Ocean engineering, 28(4), 417-438, 2001. https://dx.doi.org/10.1016/S0029-8018(00)00008-1.

9 Sutulo, S., Rodrigues, J. M., and Soares, C. G. Computation of inertial and damping characteristics of ship sections in shallow water. Ocean Engineering, 36(14), 1098-1111, 2009. https://dx.doi.org/10.1016/j.oceaneng.2009.06.013.

10 Maheri, M. R. and Severn, R. T. Experimental 


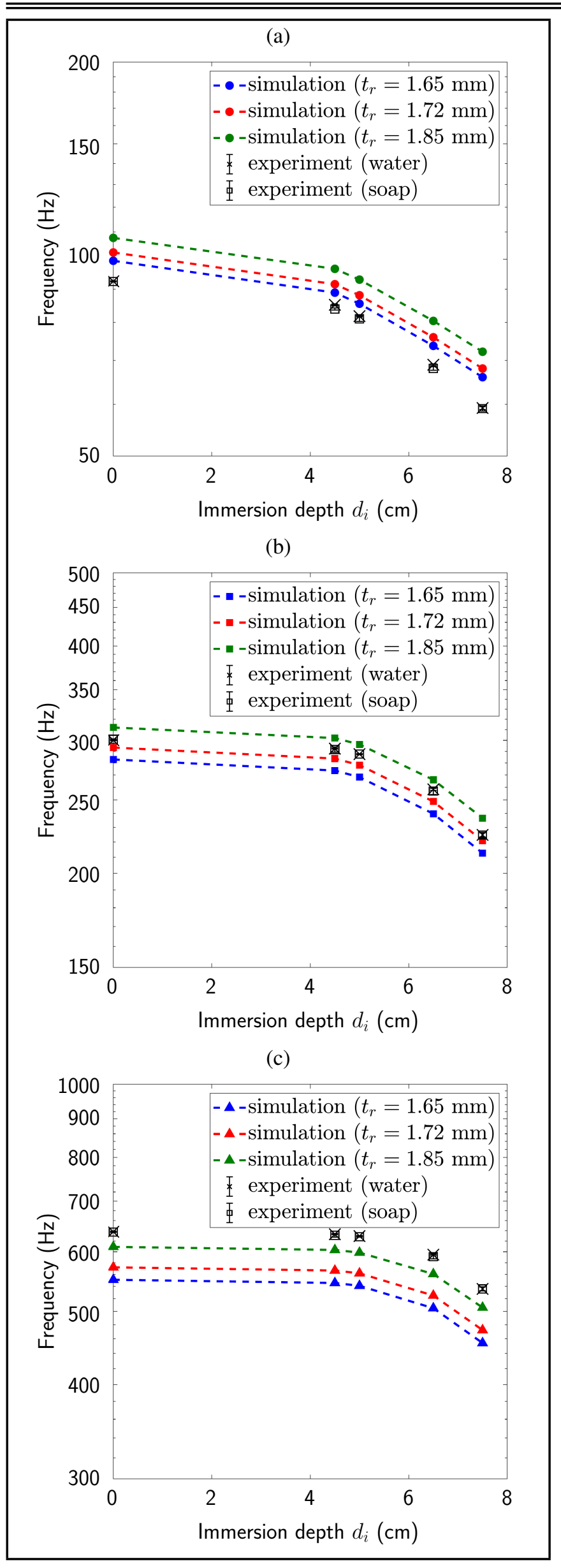

Figure 9. Wet natural frequencies of (a) mode 0C2D, (b) mode 0C3D, and (c) mode 0C4D for different values of $d_{f}$. added-mass in modal vibration of cylindrical structures. Engineering Structures, 14(3), 163-175, 1992. https://dx.doi.org/10.1016/0141-0296(92)90027-N.

11 Chang, T. P. and Liu, M. F. On the natural frequency of a rectangular isotropic plate in contact with fluid. Journal of Sound and Vibration, 236, 547-553, 2000. https://dx.doi.org/10.1006/jsvi.2000.2955.

12 Korotkin, A. I. Added masses of ship structures, volume 88. Springer Science \& Business Media, 2008. https://dx.doi.org/10.1007/978-1-4020-9432-3.

13 Arribas, F. P. and Fernández, J. A. C. Strip theories applied to the vertical motions of high speed crafts. Ocean Engineering, 33(8), 1214-1229, 2006. https://dx.doi.org/10.1016/j.oceaneng.2005.04.021.

14 Ergin, A. and Price, W. G. Dynamic characteristics of a submerged, flexible cylinder vibrating in finite water depths. Journal of Ship Research, 36, 154-167, 1992. https://dx.doi.org/SNAME-JSR-1992-36-2-154.

15 Ergin, A. and Temarel, P. Free vibration of a partially liquid-filled and submerged, horizontal cylindrical shell. Journal of Sound and vibration, 254(5), 951-965, 2002. https://dx.doi.org/10.1006/jsvi.2001.4139.

16 Yadykin, Y., Tenetov, V., and Levin, D. The added mass of a flexible plate oscillating in a fluid. Journal of Fluids and Structures, 17(1), 115-123, 2003. https://dx.doi.org/10.1016/S0889-9746(02)00100-7.

17 Ge, F., Lu, W., Wu, X., and Hong, Y. Fluidstructure interaction of submerged floating tunnel in wave field. Procedia Engineering, 4, 263-271, 2010. https://dx.doi.org/10.1016/j.proeng.2010.08.030.

18 Liu, F., Li, H., Qin, H., and Liang, B. Added mass matrix estimation of beams partially immersed in water using measured dynamic responses. Journal of Sound and Vibration, 333(20), 5004-5017, 2014. https://dx.doi.org/10.1016/j.jsv.2014.05.036.

19 Everstine, G. C. and Henderson, F. M. Coupled finite element/boundary element approach for fluidstructure interaction. The journal of the Acoustical Society of America, 87(5), 1938-1947, 1990. https://dx.doi.org/10.1121/1.399320.

20 Wang, C. D. and Meylan, M. H. A higher-ordercoupled boundary element and finite element method for the wave forcing of a floating elastic plate. Journal of Fluids and Structures, 19(4), 557-572, 2004. https://dx.doi.org/10.1016/j.jfluidstructs.2004.02.006.

21 Förster, C., Wall, W. A., and Ramm, E. Artificial added mass instabilities in sequential staggered coupling of nonlinear structures and incompressible viscous flows. Computer methods in applied mechanics and engineering, 196(7), 1278-1293, 2007. https://dx.doi.org/10.1016/j.cma.2006.09.002.

22 Van Brummelen, E. H. Partitioned iterative solution methods for fluid-structure interaction. International Journal for Numerical Methods in Fluids, 65(1-3), 3-27, 2011. https://dx.doi.org/10.1002/fld.2465. 